the performance of fluorescein di-acetate FDA vital staining, a microscopy-based test that shows viable bacilli, and Xpert threshold cycle value $(\mathrm{Ct})$ changes when assessing culture conversion at the end of the intensive phase of RR-TB treatment. Methods Between December 2015 and April 2018, we prospectively followed patients with RR-TB during the 6-month intensive phase of a 21-month standardised WHO treatment regimen. Sputum was collected and tested monthly with Auramine, FDA, Xpert MTB/RIF, and culture (Manual MGIT). Culture was considered to have converted to negative when two consecutive cultures, taken at least 30 days apart, were negative, including at least one culture between 4-6 months of treatment.

Results Forty-one patients were included in this study, $80 \%$ were male and $7 \%$ were HIV-co-infected. Conversion could not be assessed in $12(29 \%)$ patients. Among the remaining 29 patients, $9(31 \%)$ converted, and 11 (38\%) did not convert. All 9 who converted on culture had a negative FDA, and most (6) had a $\mathrm{Ct}$ trend that showed a reduction of excreted DNA (increasing Ct trend). Three of these were still positive on Auramine (excretion of dead bacilli?). Of 11 patients with positive cultures, 8 tested negative on FDA, 5 tested 'MTB not detected' on Xpert MTB/RIF, and another 2 showed a reduction of excreted DNA.

Conclusion Results from culture, FDA, and Xpert MTB/RIF provide similar results among converters but contrasting results among non-converters. Longer follow-up time is needed to assess the value of these tests to predict treatment outcome.

\section{PO 8549 ACHIEVING THE THIRD '90' AMONG KEY POPULATIONS THROUGH A DIFFERENTIATED CARE MODEL}

${ }^{1}$ Maureen Akolo*, ${ }^{2}$ Joshua Kimani, ${ }^{2}$ Larry Gelmon. ${ }^{1}$ Partners in Health and Development in Africa (PHDA), Nairobi, Kenya; ' University of Nairobi/Manitoba, Kenya

\subsection{6/bmjgh-2019-EDC.136}

Background Partners in Health and Development in Africa (PHDA) is an HIV/STI research centre that serves both female and male sex workers in Nairobi. PHDA offers HIV/STI care and treatment, and prevention services to approximately 27,400 female sex workers and 1600 men who have sex with men. Currently, the organisation has 2384 HIV-positive female and male sex workers who access HIV care and treatment services within PHDA. We realised HIV viral suppression of our clients was at 78\%, and therefore we came up with a strategy to reach at least $90 \%$ viral suppression.

Methods Patients who were virally suppressed, had their scheduled clinic visits reduced to twice annually, while viraemic clients visited the facility at least twice a month for directly observed therapy of ARVs. Clients who were non-viraemic (champions) and willing to participate in support groups were selected through simple random sampling to join the viraemic clients support groups. The champions encouraged the viraemic peers on the importance of adhering to treatment to gain viral suppression and reduce time spent at the facilities. Challenges and successes were also shared. This was done for a minimum of three consecutive months and graduation done upon viral suppression.
Results Eighty-eight per cent $(88 \% ; 2117 / 2384)$ of the total positives were bled for viral loads within the year; $78.3 \%$ (1658/2117) had viral suppression and 459/2117 had viral loads (VL) above 1000 copies per $\mathrm{ml}$ of blood. After attending the joint support groups with documented good adherence for at least three consecutive months, clients were bled for a repeat VL count, of which 84\% (386/459) had suppressed. This increased the overall viral suppression from $78.3 \%$ to $93.8 \%$ ( 2044/2177). There was $73 \%$ risk of being viraemic if not attending a support group.

Conclusion Differentiated care clients can work with viraemic clients to increase community viral load suppression.

\section{PO 8550 NATURE AND PREVALENCE OF MULTI-MORBIDITY IN FISHING COMMUNITIES ON LAKE VICTORIA, KENYA}

Zachary Kwena*, Raphael Ondondo, Catherine Makokha, Elizabeth Bukusi. Kenya Medical Research Institute, Nairobi, Kenya

\subsection{6/bmjgh-2019-EDC.137}

Introduction Multimorbidity, coexistence of two or more chronic conditions, is becoming more common across different demographic groups in sub-Saharan Africa. We investigate the nature and prevalence of multimorbidity in fishing communities on Lake Victoria in Kenya.

Methods We conducted a cross-sectional survey enrolling 679 participants in the fishing communities to establish the prevalence of HIV and non-communicable diseases (NCDs) and associated risk factors. The NCDs targeted included diabetes mellitus detected by random blood glucose (RBG) and kidney dysfuction detected by serum creatinine level and proteniuria. Hypertension was defined as systolic blood pressure $\geq 140 \mathrm{mmHg}$ and/or diastolic blood pressure $\geq 90 \mathrm{mmHg}$. Diabetes mellitus was defined as RBG $>7.8 \mathrm{mmol} / \mathrm{L}$ and renal dysfunction was defined as estimated glomerular filtration rate (eGFR) $<60 \mathrm{~mL} / \mathrm{min}$ or proteinuria. HIV was tested using rapid Determine assays and reactive results confirmed with UniGold assays. Additionally, participants were determined as having the condition if they reported being on medication for the condition. We analysed the results using descriptive statistics and used Chi-square test to discern if there were statistically significant differences by gender.

Results Overall, HIV prevalence was $36 \%$ while 15\%, 12\% and $8 \%$ of the participants were suspected to have kidney dysfuction, hypertension and diabetes mellitus, respectively. Overall, $12 \%$ of the participants had multimorbidity. The most common multimorbidity among those with any of the four chronic conditions was HIV and kidney dysfunction (29\%), followed by hypertension and kidney dysfuction (22\%), HIV and hypertension (20\%), HIV and diabetes (18\%), diabetes and kidney disease $(6 \%)$ and, lastly, diabetes and hypertension (5\%). Apart from HIV, we observed no statistically significant gender differences for any of the NCDs or various multimorbidity conditions.

Conclusion These fishing communities have a high burden of both HIV and NCDs resulting in high prevalence of different multimorbidities. 